\title{
Das Problem der praefationes
}

\author{
von Christoph Markschies ${ }^{1}$
}

Was eine praefatio ist und was sie leisten soll, scheint sonnenklar. Sie enthält alles, was benötigt wird, um eine kritische Edition benutzen zu können. Das, was ich da gerade in einem einzigen Satz formuliert habe, kann man natürlich ausführlicher sagen und ein Inventar solcher Notwendigkeiten für Benutzer von kritischen Ausgaben geben ${ }^{2}$, aber wirklich schwere Probleme scheint es beim Thema praefatio auf den ersten Blick nicht zu geben. Man könnte von daher auch mutmaßen, es gebe im wesentlichen nur eine einzige Gestalt, in der in dem Jahrhundert, das seit der Gründung der „Griechischen Christlichen Schriftsteller der ersten drei Jahrhunderte" vergangen ist, Einleitungen zu kritischen Textausgaben von ihren Editoren angelegt worden sind.

Wer sich allerdings nur ein wenig mit Editionen aus diesem Zeitraum beschäftigt hat, wird wissen, daß der Eindruck steter Gleichförmigkeit ihrer praefationes natürlich überhaupt nicht zutrifft. Ich möchte in den folgenden vierzig Minuten über diese Unterschiede sprechen und nicht nur zeigen, daß sie paradigmatisch für die grundlegenden Konzepte stehen, die mit solchen Ausgaben verbunden sind. Mir liegt außerdem daran, Ihnen den spezifischen Ort des Berliner Corpus im bunten Spektrum der Texteditionen seit 1897 ein wenig anhand jenes Problems der praefationes vorzuführen. Dabei beginne ich mit grundsätzlichen Bemerkungen zum Charakter der praefatio, behandle dann das Problem der zu verwendenden Sprache und gehe schließlich in einem dritten Abschnitt auf die Frage nach Inhalt und Länge einer Einleitung ein.

1 Den Herren Kollegen Heck und Kannicht aus Tübingen danke ich sehr herzlich für freundliche Hinweise zum Thema.

2 So beispielsweise G. Jäger, Einführung in die klassische Philologie, München ${ }^{2} 1980,54 f$. - Knapper J. Delz, Textkritik und Editionstechnik, in: F. Graf (Hg.), Einleitung in die lateinische Philologie, Stuttgart/Leipzig 1997, (51-73) 71: „Die Praefatio gibt Auskunft darüber, wie die Textgrundlage beschaffen ist ..., ob der Editor die Handschriften selbst gesammelt, kollationiert und bewertet hat oder ob er sich auf Vorgänger stützt ... Beruht die Kollation auf Autopsie, kann eine Liste der hauptsächlichen Fehlertypen ... in den wichtigsten Handschriften für einen späteren Kritiker nützlich sein. Ein Stemma sollte gezeichnet werden, wo es möglich ist ... Am Schluß steht ein Siglenverzeichnis und eine vollständige Liste der im kritischen Apparat vorkommenden Namen von modernen Autoren mit genauer Lokalisierung ihrer Beiträge“.

ZAC, vol. 8, pp. 38-58

(C) Walter de Gruyter 2004 


\section{Bemerkungen zum grundsätzlichen Charakter einer praefatio}

Meine These ist: Der Charakter einer Textausgabe wird bereits daran deutlich, wie der Editor seine praefatio anlegt - oder anders formuliert: wie er in Auseinandersetzung mit den spezifischen Vorgaben der Editionsreihe seine Einleitung anlegt. Um dies zu erkennen, ist ein Vergleich zwischen den ersten Faszikeln des Berliner und des Wiener Kirchenväter-Unternehmens lehrreich, also ein Vergleich zwischen dem Wiener Original und der Berliner Kopie, wobei sich schnell zeigen wird, daß diese Metapher das Verhältnis beider Reihen nicht präzise genug beschreibt.

Der Vergleich der beiden ersten Faszikel ist deswegen lehrreich, weil man den Anspruch auf Modernität - oder präziser: auf Modernisierung überkommener Gewohnheiten -, den die Berliner Kommission gerade auch im Blick auf das Wiener Schwesterunternehmen vertrat, an dem scheinbar ganz äußerlichen Beispiel der praefationes sehr deutlich wahrnehmen kann. Was nämlich der gewöhnliche Brauch war, von dem man sich in Berlin absetzen wollte, erkennt man an dem Bändchen, mit dem 1866 die Wiener Ausgabe startete: $\mathrm{Zu}$ den Opera des spätantiken Autors Sulpicius Severus, die Karl Halm im nämlichen Jahr als ersten Band des „Corpus Scriptorum Ecclesiasticorum Latinorum “ auf 278 Seiten edierte, wurden gerade einmal elf Seiten einer praefatio beigefügt ${ }^{3}$. Halm orientierte zu jeder Schrift des Sulpicius separat und knapp über die voraufgegangenen Editionen, berichtete über Handschriften - wobei vor dem Hintergrund heutiger Standards der Handschriftenbeschreibung ${ }^{4}$ auffällt, wie knapp seine Angaben häufig sind, die sich oft auf die reine Angabe der Handschriftensignatur beschränken. Begründungen für die Datierung einer bestimmten Handschrift fehlen praktisch vollständig. Damit entsprach Halm aber, wie gesagt, dem Standard seiner Zeit. Wiener Spezifika kann man nur an sehr wenigen Stellen entdecken: So wurde beispielsweise immer angegeben, wer für die Kollationen verantwortlich zeichnet, die beim Wiener Unternehmen bekanntlich oft nicht vom Editor ausgeführt wurden, sondern diesem vorlagen. Halm schloß sein Vorwort mit einem knappen biographischen Abriß über Sulpicius Severus, den er dem Schriftstellerkatalog des Gennadius entnahm.

Ganz anders das Berliner Unternehmen: Reichlich dreißig Jahre nach dem ersten Wiener Band orientiert die „Kirchenväter-Commission der K. Preuss. Akademie der Wissenschaften“ die Leser ihres ersten Bandes dar-

Sulpicii Severi Libri qui supersunt, rec. et commentario critico instruxit C. Halm, CSEL 1, Wien 1866, V-XIV.

4 Vgl. dazu die Angaben bei: Aristoteles graecus. Die griechischen Manuskripte des Aristoteles, untersucht und beschrieben von P. Moraux/D. Harlfinger/D. Reinsch/J. Wiesner, 1. Bd.: Alexandrien-London, Peripatoi 8, Berlin/New York 1976, XIII-XIX, und D. Harlfinger/D. Reinsch, Die Aristotelica des Parisinus Gr. 1741. Zur Überlieferung von Poetik, Rhetorik, Physiognomik, De signis, De ventorum situ, Phil. 114/115, 1970/1971, (28-50) 29f. 
über, daß ihre Ausgaben „historisch orientirende Einleitungen“ erhalten werden: „Umfangreichere Untersuchungen, die in den Prolegomenen nicht Raum haben, sollen in den von O. v. Gebhardt und A. Harnack herausgegebenen ,Texten und Untersuchungen zur Geschichte der altchristlichen Litteratur' erscheinen, deren ,Neue Folge' als ,Archiv' der Ausgabe der Schriftsteller zur Seite tritt" ${ }^{\text {}}$. Zweierlei fällt an diesen Bemerkungen auf: Die Trennung zwischen einer historisch orientierenden Einleitung einerseits und ausführlicheren Untersuchungen andererseits und der Begriff „historisch orientierende Einleitung “ an sich. Einen äußerlichen Grund für diese Konzeption einer praefatio kann man schnell angeben: Es wird schon in diesem Vorwort ganz deutlich, daß der Grund für die klare Trennung zwischen einer „historisch orientierenden Einleitung “ und „umfangreicheren Untersuchungen " zunächst einmal in der überaus komplexen überlieferungsgeschichtlichen Situation des Autors Hippolyt selbst lag, dessen Daniel-Kommentar als erster Band der Reihe erschien. „Die Herausgeber haben daher im Einverständnis mit der Commission diese Untersuchungen in das oben genannte ,Archiv' verwiesen, über alle Hauptpunkte aber in der Ausgabe selbst Rechenschaft gegeben "6. Und tatsächlich erschienen im zweiten Heft des sechzehnten Bandes der „Texte und Untersuchungen zur Geschichte der altchristlichen Literatur", zugleich dem zweiten Heft der neuen Folge, Bonwetschs „Studien zu den Kommentaren Hippolyts ${ }^{\text {"7 }}$ und Hippolytstudien von Hans Achelis ${ }^{8}$. Seit Bonwetschs Studien trug die 1882 begonnene Serie auch den bekannten Untertitel „Archiv für die von der Kirchenväter-Commission der Kgl. Preußischen Akademie der Wissenschaften unternommene Ausgabe der älteren christlichen Schriftsteller". Während Bonwetsch in seinem Appendix zu den Prolegomena der Ausgabe vor allem Fragen der Komposition verhandelte und ausführlich seine Entscheidung für eine Datierung des Daniel- wie des Hoheliedkommentars begründete ${ }^{9}$, legte Achelis ausführliche Untersuchungen zum Autor Hippolyt vor, besprach die antiken Nachrichten über den Autor und trug zu den Fragmenten ausführliche Handschriftenbeschreibungen nach, aus denen seine Vertrautheit mit dem Katenenmaterial erkennbar ist. In der Ausgabe der kleinen Hippolyt-Fragmente durch Achelis, dem zweiten Halbband der Reihe GCS, finden sich in den „Vorbemerkungen“ nur ganz wenige Zeilen pro Textstück und unmittelbar vor der Edition eine Zusammenstellung der verwendeten Siglen.

\footnotetext{
Hippolytus Werke, Bd. 1 Exegetische und homiletische Schriften, hg. ... v. G.N. Bonwetsch u. H. Achelis, GCS Hippolyt I, Leipzig 1897, unpaginiertes Vorsatzblatt vor dem Innentitel, fol. $0^{\mathrm{v}}$.

6 Ebd.

7 G.N. Bonwetsch, Studien zu den Kommentaren Hippolyts zum Buche Daniel und Hohen Liede, TU 16/2 = NF 1/2, Leipzig 1897.

8 H. Achelis, Hippolytstudien, TU 16/4 = NF 1/4, Leipzig 1897.

9 Bonwetsch, Studien (wie Anm. 7), 81-85; vgl. seine Ausgabe, GCS Hippolyt I, XX.
} 
Interessanter als diese pragmatische Trennung von knapper Einleitung und ausführlicher Untersuchung scheint mir der Begriff „historisch orientierende Einleitung “, den die Kommission im Vorwort zum ersten Band und zugleich zur ganzen Reihe verwendet. Es ist wohl nicht zu spitzfindig, in dieser Formulierung einen Gegensatz mitzuhören, der eine „historisch orientierende " Einleitung von einer primär philologisch orientierenden Einleitung abhebt. Mir scheint, daß damit die Berliner mindestens implizit einen gewissen Gegensatz zu den Wienern markiert haben, wenn auch keinen strengen Unterschied. Warum das Berliner Unternehmen solches Gewicht auf die historische Dimension in den Einleitungen legte, kann man vergleichsweise leicht klarmachen: Der Initiator Harnack verband mit dem Unternehmen ja von vornherein weniger philologische als vielmehr historische Ziele, wie Walther Eltester anläßlich des fünfundsiebzigjährigen Jubiläums ausführlicher gezeigt hat ${ }^{10}$ : Die „paläontologische Schicht“ des Christentums sollte erhellt und präzise rekonstruiert werden. Fragt man nach unmittelbaren Vorbildern der Berliner Entscheidung für „historisch orientierende Einleitungen ", so wird man schnell auf die große Edition „The Apostolic Fathers“ des Durhamer Bischofs Joseph Barber Lightfoot aufmerksam. 1890, kurz vor dem Tod des Autors, war die zweite Auflage der gleichnamigen monumentalen fünfbändigen Gesamtausgabe abgeschlossen. Der Edition geht ein umfangreicher einleitender Band voraus, der die Inhalte einer traditionellen praefatio mit ausführlichen Exkursen kombiniert ${ }^{11}$. Harnack, der ja selbst auf dem Gebiet der Edition der sogenannten „Apostolischen Väter“ tätig war, rühmte die erste Auflage: „We may say, without exaggeration, that this work is the most learned and careful patristic monograph which has appeared in the nineteenth century"12. Schon wegen solcher Urteile, natürlich aber auch wegen der deutlichen Bezüge auf Lightfoot in Harnacks sonstigem Werk - man denke nur an die „Chronologie“"13, scheint es mir nicht vermessen, dieses Werk als Vorbild für die besondere Gestalt der Berliner praefationes zu postulieren.

10 W. Eltester, Zur Geschichte der Berliner Kirchenväterkommission anläßlich der 75 . Wiederkehr ihres Gründungsjahres, ThLZ 93, 1968, (11-19) 12, und Ch. Markschies, Einleitung, in: ders./St. Rebenich (Hgg.), Adolf von Harnack, Protokollbuch der Kirchenväter-Kommission der Preußischen Akademie der Wissenschaften 1897-1928, Berlin/New York 2000, (1-9) 4.

11 The Apostolic Fathers, Part II S. Ignatius, S. Polycarp, Revised Texts with Introductions, Notes, Dissertations, and Translations, by J.B. Lightfoot, Vol. I, London/New York 1889.

12 A. Harnack, Rez. Lightfoot, The Apostolic Fathers Bd. II/1-3, London 1885, The Expositor $3^{\text {rd }}$ ser. Vol. II, London 1885, (401-414) 401. Vgl. ders., Rez. E. Hatch, Essays in Biblical Greek, Oxford 1889, in: ThLZ 15, 1890, (297-301) 297: „Lightfoot war der gelehrteste und würdigste Repräsentant der conservativen kritischen Schule in England.“ und M. Hengel, Bischof Lightfoot und die Tübinger Schule, ThB 23, 1992, (5-33) 1012.

13 Hengel, Bischof Lightfoot (wie Anm. 12), 25. 
Mir scheint allerdings trotz dieses unmittelbaren Vorbildes, daß vor dem Hintergrund der Situation des Jahres 1897 die Ankündigung, im Berliner Kirchenväterkorpus „historisch orientierende“ praefationes zu veröffentlichen, tatsächlich auch ein Zeugnis eines beachtlichen Modernisierungsimpetus darstellte. Das Unternehmen hat damit durchaus Schule gemacht, ohne die Dominanz einer primär "philologisch orientierenden“ Einleitung vollständig beenden zu können. Ich erlaube mir, dies zu demonstrieren, indem ich kurz zum Vergleich jeweils frühe Faszikel anderer bekannter Editionsreihen von spätantiken Texten heranziehe, die nach 1897 erschienen sind: Eine philologisch orientierende Einleitung im ganz strengen Sinne bot der erste Band des „Corpus Christianorum“, dessen lateinische Series 1954 zu erscheinen begonnen hat und als neuer Migne zunächst durchaus auch korrigierte Abdrucke von anderwärts edierten Texten bot. Hier folgte man eindeutig der Tradition einer primär philologisch orientierenden praefatio: Wenige Zeilen über den Autor Tertullian, dem die ersten beiden Bände der Reihe gewidmet sind, einige Bemerkungen über die mittelalterlichen Sammlungen seiner Werke, ein Stemma und eine instruktive Tabelle über die indirekte Überlieferung auf der Basis einer Untersuchung von Harnack; das ist schon nahezu alles, was die erste praefatio dieser Reihe bietet ${ }^{14}$.

Ganz anders dagegen die „Sources Chrétiennes“, eine Reihe, die freilich von Anfang an durchaus auch einen theologischen Impetus verfolgte bekanntlich versuchte man vor dem Hintergrund der „nouvelle théologie“ durch Übersetzungen und Kommentierung von Vätertexten die Dominanz der neuscholastischen Theologie im Bereich der römisch-katholischen Konfessionstheologie zu brechen und eine - wie man heute sagen müßte plurale theologische Denkwelt gegen den traditionellen Monismus zu set$z{ }^{15}$. Es verwundert nicht, daß die praefationes des Unternehmens von dieser Zielsetzung, die über eine reine Philologie deutlich hinausweist, erkennbar geprägt sind: Gustave Bardy stellte seiner Übersetzung der Supplicatio des Athenagoras, die als drittes Heft in den „Sources Chrétiennes" im Jahre 1943 erschien, eine umfangreiche Einleitung voran, die fast ein Drittel des ganzen Buches ausmacht ${ }^{16}$. Obwohl die Reihe damals noch gar keine Editionen enthielt, orientierte er nicht nur über Person und Werk des Athenagoras, Inhalt und Gattung des Werkes, sondern auch über

14 Quinti Septimi Florentis Tertulliani Opera, Pars I Opera Catholica. Adversus Marcionem, Turnhout 1954, V-XXVII; vgl. A. Harnack, Tertullian in der Litteratur der alten Kirche, SPAW.PH 1895, (545-579) 562 = ders., Kleine Schriften zur Alten Kirche. Berliner Akademieschriften 1890-1907, mit einem Vorwort v. J. Dummer, Opuscula IX/1, Leipzig 1980, (247-281) 264.

15 É. Fouilloux, La collection „Sources Chrétiennes“. Éditer les Pères de l'Église au XX siècle, Paris 1995, 12-24.115-120.

16 Athénagore, Supplique au sujet des Chrétiens. Introduction et traduction de G. Bardy, SC 3, Paris/Lyon 1943, 7-67. 
„La doctrine d'Athénagore“ 17 und schließlich knapp über „Manuscrits, éditions, traductions“"18. 1949 erschien eine zweite Auflage des zweiten Bandes der Reihe; Claude Mondésert hatte seiner kommentierten Übersetzung des Protrepticus des Clemens von Alexandria nun einen griechischen Lesetext auf der Basis der Berliner Edition hinzugefügt und führte in diese Rezension auf wenigen Seiten ein ${ }^{19}$. Das in diesen beiden genannten Bänden repräsentierte Verhältnis von einer ausführlichen Einleitung in Person, Werk und Theologie eines Autors samt knappen Bemerkungen zur Textrezension scheint mir über lange Jahre für die französische Reihe repräsentativ gewesen zu sein - man könnte vielleicht von einer theologisch orientierten, an Theologen als ersten Leserkreis gerichteten Einleitung sprechen und diese Form der praefatio der mehr philologisch orientierenden im Wiener Corpus und der mehr historisch orientierenden im Berliner Corpus gegenüberstellen.

In Klammern gesagt: Ich habe von den jeweils ersten Bänden der verschiedenen Editionsreihen gesprochen; daß es jeweils eine ganze Reihe von Ausnahmen vom Prinzip gibt, beispielsweise rein philologisch orientierte praefationes im Berliner Corpus, wird uns noch beschäftigen. Man muß sich dabei klarmachen, daß antike christliche Literatur durchaus eine Reihe von gemeinsamen Überlieferungs- und Editionsproblemen aufweist, die sich in der klassischen Literatur so nicht stellen - man denke allein an die Fülle von Handschriften, die das Neue Testament oder Schriften des Johannes Chrysostomus bezeugen: Kurt Treu hat vor längerer Zeit solche einschlägigen Besonderheiten patristischer Editionen klar analysiert ${ }^{20}$. Mit den drei Stichworten „philologisch“, „historisch“ und „theologisch“ befinden wir uns natürlich nach wie vor im Raum der klassischen Universität und ihrer Gliederung in Disziplinen. Diesen Raum verlassen wir, wenn wir uns nun in einem zweiten Abschnitt der Frage zuwenden, in welcher Sprache praefationes verfaßt worden sind.

\section{Bemerkungen zur sprachlichen Gestalt einer praefatio}

Wenn wir unseren Ausgangspunkt wieder bei einem Vergleich zwischen dem Wiener und dem Berliner Kirchenväter-Corpus nehmen, wird auch hier schnell deutlich, inwiefern man sich in Berlin um Modernität bemühte. Während bis zum heutigen Tage Wiener praefationes in lateinischer Sprache abgefaßt sind und entsprechend auch der textkritische Apparat mit

7 Ebd. 44-65.

18 Ebd. 65-69.

19 Clément d'Alexandrie, Le Protreptique. Introduction, traduction et notes de C. Mondésert, SC 2, Paris ${ }^{2} 1949$, 45-50.

20 K. Treu, Überlieferungs- und Editionsprobleme der griechischen Patristik, SEÅ 34, 1969, 186-200 = D. Harlfinger (Hg.), Griechische Kodikologie und Textüberlieferung, Darmstadt 1980, (613-628) 618-620. 
lateinischen Siglen arbeitet ${ }^{21}$, setzte man in Berlin von Anfang an auf die deutsche Sprache - wenn man sich an die heftigen Debatten erinnert, die über die Bedeutung der klassischen Sprachen für die Schulerziehung im Kaiserreich ausgefochten wurden und an denen sich Adolf von Harnack durchaus beteiligte, dann wird die forschungspolitische Brisanz einer solchen Entscheidung deutlich. Das bereits erwähnte Vorwort zur Reihe im ersten Band geht freilich auf das Problem, das mit der Wahl der deutschen Sprache verbunden war, mit keiner Silbe ein. Die äußerst sparsame öffentliche Kommentierung eines revolutionären Aktes fällt vor allem dann auf, wenn man sie mit der teilweise erregten Reaktion auf die SophoklesAusgabe vergleicht, die Hugh Lloyd-Jones und Nigel G. Wilson 1990 im Rahmen der traditionellen Oxforder „Scriptorum classicorum bibliotheca Oxoniensis“ veröffentlichten. Wenn auch noch Titelblatt und Sigla dieses Bandes im vertrauten Latein abgefaßt sind, wurden die Leser doch zu Beginn durch eine englische Einleitung überrascht - weswegen sich die Editoren offenbar bemüßigt fühlten, ihre Wahl der Landessprache mit zwei Argumenten zu begründen, nämlich der Möglichkeit, Handschriften in englischer Sprache präziser zu beschreiben und auf diese Weise auch einem größeren Leserkreis das Verständnis der praefatio zu erleichtern ${ }^{22}$. Ansonsten entsprach das Vorwort dem traditionellen philologisch orientierenden Stil der Oxforder Vorworte; so lehnten es die Herausgeber beispielsweise explizit ab, in ihrer Einleitung eine „short biography of the poet“ $\mathrm{zu}$ geben $^{23}$. Morris L. West kommentierte in einer nicht unfreundlichen Rezension des Bandes durchaus doppeldeutig: „And an OCT with a preface in English! This is the end of civilization as we have known it“24. Andere Rezensenten wählten da bescheideneres Vokabular, wenn sie die Gründe der beiden Altmeister „sensible“ nannten, „even if they evoke a certain sadness" 25 . Daß freilich damit ein "Novum in Ausgaben unseres Faches“" zu konstatieren gewesen sei, wie Bernhard Zimmermann im „Gnomon“ behauptete, wird man angesichts der fast hundert Jahre alten Praxis der „Griechischen Christlichen Schriftsteller" kaum nachvollziehen können ${ }^{26}$.

Bei dem Berliner Corpus, das im Titel der berühmten Denkschrift Harnacks vom Oktober 1893 noch relativ unbestimmt „Ausgabe der vornicänischen Väter“ oder (im Text) „Ausgabe der gesamten vornicä-

21 Vgl. dazu aus den Richtlinien für die Editoren der Bibliotheca Teubneriana: „Die Sprache der BT ist das Lateinische. Grammatische Korrektheit und ein sachlicher, klarer Stil sind die Voraussetzungen für die Benutzbarkeit jeder Ausgabe“ (S. 1; undatierte Kopie aus den sechziger Jahren).

22 Sophoclis Fabulae, recognoverunt brevique adnotatione critica instruxerunt H. LloydJones et N.G. Wilson, SCBO, Oxford 1990, V-XVI.

23 Ebd., XIV.

24 M.L. West, The new OCT of Sophocles, ClR 41, 1991, (299-301) 301.

25 R. Renehan, The new Oxford Sophocles, CP 87, 1992, (335-375) 337; ähnlich nüchtern J. Jouanna in REG 104, 1991, (291f.) 292.

26 B. Zimmermann, Rez. H. Lloyd-Jones/N.G. Wilson, Gn. 65, 1993, (100-109) 100. 
nischen griechischen Literatur" heißt, weil beispielsweise auch die ,vornizänischen Konzilien' in einem Band veröffentlicht werden sollten, ist wenn ich recht sehe - in einem einzigen Fall die deutsche Sprache, die man in den praefationes verwendete, zum Thema einer regelrechten literarischen Auseinandersetzung geworden. Anläßlich dieser Auseinandersetzung in den Jahren 1910/1911 äußerte sich Harnack auch über die Motive, von Anfang an konsequent die deutsche Sprache zugrundezulegen und nicht die lateinische. Freilich ist das eine späte und nicht unproblematische Sinndeutung ex post. Noch 1893 hatte Harnack in einem Brief an Gebhardt und Loofs das geplante Werk mit dem lateinischen Titel, mit dem er es im Januar 1891 bei der Akademie beantragt hatte, „Corpus scriptorum Graecorum Antenicaenorum“ genannt und schrieb unter Datum vom 8.12.1893:

\begin{abstract}
„Das ,Corpus scriptorum Graecorum Antenicaenorum“ wird wohl deutsch erscheinen. Der Buchhändler sagt, deutsch sei eher günstiger als lateinisch, jedenfalls nicht ungünstiger. Die ersten Mitarbeiter, Bonwetsch + Achelis, haben mich ausdrücklich um Deutsch gebeten, und manche von denen, die mitarbeiten werden, können kein Latein schreiben. Auch die Engländer schreiben ja jetzt fast regelmäßig englisch. Ich bitte, daß Sie beide sich darüber äußern, ob sie etwas gegen ,deutsch“ einzuwenden haben. Diels verlangt, wenn deutsch edirt wird (wogegen er nicht ist), daß auch der Apparat deutsch edirt wird u. ich muß ihm Recht geben. Sind die Herren, wie ich hoffe, auch für deutsch, so bitte ich, über einen passenden Gesamttitel nachzudenken. Es ist eine schwierige Sache. Herzlich grüßend, A. Harnack“27.
\end{abstract}

Man gewinnt angesichts dieser Äußerungen den Eindruck, daß die Entscheidung, Einleitung und Apparat in deutscher Sprache abzufassen, 1893 keineswegs primär als bewußter Akt der Modernisierung interpretiert werden darf, sondern einerseits ökonomischer Notwendigkeit gehorchte und andererseits mit der ungewöhnlich hohen Zahl von Nichtphilologen begründet war, die das Unternehmen trugen. Die Philologen nannten das Werk ohnehin gern „Corpus Berolinense“.

Überraschenderweise wird in den ersten Jahren des Berliner Unternehmens nun nicht etwa die Entscheidung, eine deutsche praefatio der Edition voranzustellen, von Philologen kritisiert oder auf die merkwürdige Inkonsequenz aufmerksam gemacht, griechische Texte ohne eine deutsche Übersetzung zu publizieren. Vielmehr wird die Nötigung zur deutschen Sprache im Zeitalter des allgemeinen nationalistischen Überschwangs von französischen Gelehrten angegriffen: Als 1911 in den „Comptes rendues“ der „Académie des Inscriptions et Belles-Lettres “28 die Verwendung der deut-

27 Brief A. Harnack an O. v. Gebhardt und F. Loofs vom 8.12.1893.

28 Comptes rendues, November 1911, 679: „Une chose est à regretter dans l'œuvre considérable de M. Parmentier, c'est que la Kirchenväter-Commission de Berlin lui ait imposé d'écrire cent pages de prolégomènes dans un idiome qui n'est ni sa langue maternelle propre ni la langue internationale de l'érudition. Ce n'est pas lui qui répond de cet abus". 
schen Sprache in der Theodoret-Ausgabe von Léon Parmentier bemängelt wurde $^{29}$, replizierte Harnack in vergleichsweise deutlicher Form. Freilich unterscheidet sich seine Rekapitulation der ursprünglichen Entscheidungsprozesse im Jahr 1911 doch stark von dem, was er 1893 selbst geschrieben hatte. Auf den Vorwurf, Parmentier sei zur Verwendung einer Sprache gezwungen worden, die weder seine Muttersprache sei noch „la langue internationale de l'érudition“, schrieb Harnack in den Sitzungsberichten:

„Die Kirchenväter-Commission hat, als sie ihre Aufgabe übernahm, eingehend
erwogen, in welcher Sprache die Ausgabe erscheinen solle. Die Entscheidung fiel
zugunsten der deutschen Sprache aus, weil eingezogene Erkundigungen ergaben,
daß Engländer, Amerikaner, Skandinavier, Russen und Griechen die deutsche
Sprache der lateinischen vorziehen. Dieser Tatsache gegenüber mußte die Rück-
sicht auf die romanischen Völker zurücktreten, zumal da vorauszusehen war,
daß der Absatz der Ausgabe bei diesen erheblich geringer sein würde als bei
jenen. Dieser Voraussicht hat der Erfolg recht gegeben. Nach Mitteilung des
Herrn Verlegers gehen nach England und Amerika etwa 90 Exemplare, nach
Frankreich 20. Der Kirchenväter-Commission gereicht es zu besonderer Freude,
daß an ihrer Ausgabe nicht nur Deutsche, sondern auch Belgier, Finnländer und
Amerikaner mitarbeiten; aber die Einheit der Ausgabe würde gesprengt werden,
wenn die Kommission jedem gestatten würde, in seiner Sprache zu schreiben,
und es ist auch keinem Mitarbeiter eine solche Forderung gestellt worden“30.

Harnack verwies im Fortgang nicht nur auf die „Sitzungsberichte“ der Akademie samt den „Texten und Untersuchungen“ des Unternehmens, die selbstverständlich fremdsprachigen Publikationen offenstünden, sondern spielte implizit auf den durchgängig lateinischen Charakter des Wiener Parallelunternehmens an (,ein Corpus scriptorum kann aber nur in ein und demselben Gewande erscheinen“). Es gebe „keine alleinherrschende ,langue internationale de l'érudition“ mehr"; zudem hätte schon vor dreißig Jahren Lightfoot seine Ausgabe der apostolischen Väter in englischer Sprache veröffentlicht ${ }^{31}$, Bidez seine Edition des Euagrius mit einer englischen Einleitung ${ }^{32}$ und Boehmer die Chronica fratris Jordani mit einer französischen Einleitung publiziert ${ }^{33}$.

Vergleicht man Harnacks Rückblick auf die Gründungsphase knapp fünfzehn Jahre nach den entscheidenden konzeptionellen Weichenstellungen einerseits und die wenigen erhaltenen Dokumente aus der Gründungsphase andererseits, dann verwundert die energische sachliche Begründung für eine mehr pragmatische (oder soll man gar sagen: ökonomische) Ent-

29 Theodoret, Kirchengeschichte, hg. von L. Parmentier, GCS 19, Leipzig 1911.

30 SPAW.PH 1913 (Sitzung vom 23. Januar 1913), ebd. 143f.

31 The Apostolic Fathers. A revised Text with Introductions, Notes, Dissertations, and Translations by J.B. Lightfoot, 5 Bde., Hildesheim 1973 (= London 1889-1890).

32 The Ecclesiastical History of Evagrius with the Scholia ed. with Introduction, Critical Notes, and Indices by J. Bidez, Amsterdam 1964 (= London 1898).

33 Chronica Fratris Jordani, ed., notis et commentario ill. H. Boehmer, Collection d'études et de documents sur l'histoire religieuse et littéraire du Moyen âge 6, Paris 1908. 
scheidung schon ein wenig, sie dürfte den politischen Umständen geschuldet gewesen sein, vor deren Hintergrund man die Kritik des französischen Kollegen wahrnehmen muß. In dieser Hinsicht ist, wie vor einiger Zeit gezeigt wurde, die Affäre um die Sprache der praefationes am Vorabend des ersten Weltkrieges allerdings für die französische Wahrnehmung deutscher Patristik keineswegs charakteristisch ${ }^{34}$. Abschließen möchte ich diesen zweiten Abschnitt mit dem Hinweis darauf, daß auch diese Regel, deutsche praefationes und Apparate anzufertigen, in Berlin durchbrochen wurde, so wie wir das bei der Verpflichtung auf eine historisch orientierende praefatio sahen: Erik Tidner versah im Jahre 1963 seine Ausgabe von Kirchenordnungstexten aus dem Codex Veronensis LV (53), darunter der Didascalia Apostolorum, mit einem lateinischen Vorwort und legte auch den Apparat in dieser Sprache an, während sein sprachlicher Kommentar zu diesem Text 1938 deutsch publiziert worden war $^{35}$; eine Tatsache, die nochmals die wesentlich pragmatische Tendenz der Berliner Kommission dokumentiert.

Für einen dritten und letzten Abschnitt kommen wir auf die eingangs geäußerte Vermutung zurück, daß es im wesentlichen selbstverständlich sei, was in einer praefatio zu erwarten ist, und von daher mit einer gewissen Gleichförmigkeit von praefationes zu rechnen sei, auch und gerade beim Berliner Kirchenväter-Unternehmen.

\section{Bemerkungen zu Inhalt und Länge einer praefatio}

Ein Blick auf die vielen praefationes, die sich seit über hundert Jahren in den Bänden der Berliner Kirchenväterausgabe befinden, zeigt freilich das schlichte Gegenteil von Gleichförmigkeit, nämlich beträchtliche Unterschiede. Das lag ganz gewiß nicht an den mangelnden Vorgaben für die diversen Editoren. Spätestens seit den fünfziger Jahren war von der Kommission für alle ihre festen wie freien Mitarbeiter schriftlich normiert, was eine Einleitung zu enthalten habe, um den Veröffentlichungen in formaler Hinsicht die Einheitlichkeit zu sichern. Trotzdem differieren die einzelnen praefationes doch erheblich nach Stil, Inhalt und Länge. Die Vorgaben verlangten (und verlangen bis heute) von den Editoren, in einer praefatio sechs Punkte zu behandeln ${ }^{36}$ :

34 J. Fugmann/K. Pollmann, Wissenschaftsgeschichtliche Tendenzen zwischen Frankreich und Deutschland in der Patristik von 1870-1930 am Beispiel von Rezensionen, in: J. Fontaine/R. Herzog/K. Pollmann (Edd.), Patristique et Antiquité tardive en Allemagne et en France de 1870 à 1930. Influences et échanges. Actes du Colloque franco-allemand de Chantilly (25-27 octobre 1991), EAug 27, Paris 1993, (239-271) 245.

35 Didascaliae Apostolorum. Canonum Ecclesiasticorum. Traditiones apostolicae versiones latinae, recensuit E. Tidner, TU 75, Berlin 1963; ders., Sprachlicher Kommentar zur lateinischen Didascalia Apostolorum, VHAAH 45/1, Stockholm 1938.

36 Nach den Richtlinien der Kommission, wie sie bis 1999 gültig waren. 
- Zeugnisse des Altertums über den Autor und seine Schriften

- Angabe und Beschreibung der Handschriften

- Abschätzung ihres kritischen Wertes und Bezeichnung ihres gegenseitigen Verhältnisses

- Aufzählung und Wertung der indirekten Überlieferungsquellen

- Aufzählung und Würdigung der früheren Ausgaben

- Mitteilung über die Dauer der Vorarbeiten und des Druckes.

Vergleicht man diesen Katalog mit anderen Aufstellungen dieser Art, so gehört er durchaus zu den umfangreicheren Kriterienkatalogen, wie sich etwa durch einen Vergleich mit den Regeln der „Collection Budé“ deutlich machen ließe: In Berlin fordert man ausführliche Bemerkungen zum Autor eines Textes, die französische Reihe verlangt dies nicht ${ }^{37}$. Sucht man nach einer Rechtfertigung für die Berliner Ausführlichkeit, so kann man neben der allgemeinen historischen Einleitung mit Kurt Treu auf besondere „Schwierigkeiten patristischer Editionen“ hinweisen:

„Die Überlieferungsgeschichte ist oft komplizierter als bei klassischen Texten, deren Wortlaut oft schon von der antiken Philologie normiert worden ist. Die Zahl der Handschriften ist größer, insbesondere bei den Autoren der Hochpatristik und bei Werken, die in die liturgisch-hagiographische Tradition eingegangen sind. Offene Überlieferung mit Quereinflüssen ist häufig, Zusammenfassung von Familien oft erschwert, Aufstellung von Stammbäumen nicht selten unmöglich. Sekundärüberlieferungen in Katenen und Florilegien, Parallelüberlieferung in freieren Bearbeitungen mit Verkürzungen und Erweiterungen erschweren die Arbeit und die Darbietung. Für orientalische Übersetzungen sind nicht viele Editoren selbst kompetent, oft muß Gemeinschaftsarbeit organisiert werden. Die Einarbeitung in Sprache, Stil und Gedankenwelt der Autoren erfordert oft beträchtlichen zusätzlichen Arbeitsaufwand, auch bei guter klassischer Sprachkenntnis des Editors. Bei der starken Traditionsbestimmtheit der spätantiken Literatur allgemein und der christlichen im besonderen steckt in dem Apparat der Quellen und Testimonien gewöhnlich mehr an Arbeit und kritischem Urteil, als ins Auge fällt" ${ }^{38}$.

37 Règles et recommandations pour les éditions critiques (Série latine), CUFr, Paris 1972. - Vgl. dazu aus den „Richtlinien für die Editoren der Bibliotheca Teubneriana“: „Die Praefatio beschreibt sämtliche handschriftlichen Zeugen in knapper, aber ausreichend informierender Form; stellt das Verhältnis der Zeugen zueinander durch einige charakteristische Sonderfehler klar; illustriert das Verhältnis durch ein Stemma; behandelt die Eigenart und den Wert des Archetyps und der Hyparchetypi; bezieht die indirekte Überlieferung in die Skizze der Textgeschichte ein; behandelt Orthographisches und Dialektisches; gibt Beispiele für inhaltliche und stilistische Gesichtspunkte bei der Textkonstitution. Literarische und historische Probleme behandelt die Praefatio nicht; nur bei Erstausgaben, Fragment- und Exzerptsammlungen ist eine Einführung in die literarischen Fragen angebracht. Testimonia zu Autor und Werk können an den Schluß der Praefatio gestellt werden" (S. 1f. - undatierte Kopie aus den sechziger Jahren).

38 K. Treu, Das Korpus der "Griechischen Christlichen Schriftsteller“ im Rahmen der Gegenwartsentwicklung der Patristik, in: J. Irmscher/K. Treu (Hgg.), Das Korpus der Griechischen Christlichen Schriftsteller. Historie, Gegenwart, Zukunft, TU 120, Berlin 1977, (7-13) 9f. 
Zum Genre derartiger Vorgaben für die Abfassung einer praefatio gehört natürlich auch der Hinweis auf die Dinge, die dort nicht hineingehören. Der Berliner Katalog nennt „eingehendere Untersuchungen ... zur Feststellung der Verwandtschaft der einzelnen Handschriften zueinander" und solche über „Echtheitsfragen und andere literaturgeschichtliche Probleme“, die Regel der französischen Reihe, an der sich auch die „Sources Chrétiennes“ orientiert haben ${ }^{39}$, schließen aus, was nicht im strengen Sinne zur Interpretation des Textes beiträgt, und nennen systematische Bemerkungen zur Grammatik, zum Stil, zur Metrik oder zu den Klauseln ${ }^{40}$.

Wenn man an diesen Punkten entlanggeht und Berliner Ausgaben aus hundert Jahren Revue passieren läßt, zeigen sich schnell die großen Unterschiede. Einige Herausgeber haben alle Ausführungen in die „Texte und Untersuchungen" verbannt und beschränken sich auf wenige Seiten in der Ausgabe, so beispielsweise Erich Klostermann im ersten Band zum Matthäus-Kommentar des Origenes ${ }^{41}$. Die meisten Editoren legten die von der Kommission einst gewünschte historisch orientierende Einleitung vor, aber längst nicht alle. Es gibt auch rein philologisch orientierte und orientierende praefationes: Der 1929 verstorbene Berliner Philologe Wilhelm Baehrens ergänzte reichlich zweihundert Seiten der lateinischen Übersetzung von Arbeiten des Origenes zum Hohen Lied mit gerade einmal vierzehn Seiten Vorwort zu den Handschriften und einer Seite zur „Bücherzahl (und Textgeschichte)“, dabei eine längere Monographie in den „Texten und Untersuchungen" zusammenfassend ${ }^{42}$. Albert Siegmund hat dann ziemlich schroff erklärt, Baehrens habe dabei ,allzuschnell Ueberlieferungsgeschichte zusammengeklittert" ${ }^{\text {“43 }}$; die Herausgeber der Werke in den "Sources Chrétiennes" scheint das nicht davon abgehalten zu haben, Baehrens' Text weitgehend zu folgen und lediglich summarisch auf seine Rekonstruktion der Überlieferungsgeschichte hinzuweisen ${ }^{44}$. Molly Whittaker versah ihre

39 Fouilloux, La collection „Sources Chrétiennes“ (wie Anm. 15), 147.

40 Règles et recommandations (wie Anm. 37), 3.

41 Origenes Werke, Bd. 10 Origenes, Matthäuserklärung, die griechisch erhaltenen Tomoi hg. unter Mitwirkung v. E. Benz v. E. Klostermann, GCS Origenes X, Leipzig 1935, VIIXIII, mit Verweis auf E. Klostermann/E. Benz, Zur Überlieferung der Matthäuserklärung des Origenes, TU 47/2, Leipzig 1931, und E. Klostermann, Nachlese zur Überlieferung der Matthäuserklärung des Origenes, TU 47/4, Leipzig 1932.

42 Origenes Werke, Bd. 8 Homilien zu Samuel I, zum Hohelied und zu den Propheten. Kommentar zum Hohelied in Rufins und Hieronymus' Übersetzungen, hg. v. W.A. Baehrens, GCS Origenes VIII, Leipzig 1925, XIV-XXVII. 26-241; vgl. aber ders., Überlieferung und Textgeschichte der lateinisch erhaltenen Origeneshomilien zum Alten Testament, TU 42/1, Leipzig 1916, 131-158. - Eine allgemeine Übersicht bei J. Irmscher, L'edizione di Origene nei „Griechische Christliche Schriftsteller“, Aug. 26, 1986, 575-578.

43 A. Siegmund, Die Überlieferung der griechischen christlichen Literatur in der lateinischen Kirche bis zum zwölften Jahrhundert, ABBA 5, München-Pasing 1949, 122 Anm. 1 mit Begründung ebd.

44 „Nous reproduisons à peu de choses près le texte de l'édition de W.A. Baehrens au tome VIII des Origenes Werke de la collection berlinoise G.C.S. “: Origène, Commentaire sur le Cantique des Cantiques. Tome I Texte de la version latine de Rufin, Introduction, 
lang erwünschte Ausgabe des „Hirten des Hermas“ mit einem kurzen, rein philologisch orientierenden und orientierten Vorwort ${ }^{45}$. Die Kommission erklärte nicht, warum sie den Band mit dem Reihentitel „GCS Apostolische Väter I" versehen hat, man findet zudem auch keinerlei Angaben zur antiken christlichen Tradition über den Autor „Hermas“ und die vielfältigen Thesen zur Datierung des Werkes bzw. seiner literarischen Schichtung. Henry Chadwick trug diese Informationen in seiner Rezension des Bandes in gewohnt dezenter Weise nach ${ }^{46}$.

$\mathrm{Zu}$ den rein philologisch orientierten praefationes im Rahmen der „Griechischen Christlichen Schriftsteller" muß man wohl auch das Vorwort von Bernhard Rehm zu den pseudoklementinischen Rekognitionen in Rufins Übersetzung rechnen, aus dem man zwar viel über Handschriften, aber nichts zu den komplizierten literarkritischen und theologiegeschichtlichen Fragen im Zusammenhang mit diesem Werk erfährt ${ }^{47}$. Ebenso zählt zu dieser Gruppe die praefatio von Emmanuel Amand de Mendieta und Stig Y. Rudberg zu den Hexaemeron-Homilien des Basilius von Caesarea, die durch eine ausführliche Untersuchung zur Handschriftentradition in den „Texten und Untersuchungen" ergänzt wurde ${ }^{48}$. Man kann mit aller Zurückhaltung sagen, daß sich so orientierte Vorworte seit 1950 häufiger als zuvor in der Berliner Reihe finden. Umgekehrt gibt es auch Beispiele für historisch orientierende Einleitungen, die ein Philologe mit tiefer Enttäuschung lesen muß, weil elementare Informationen fehlen: Rudolf Riedinger verweist in seiner Einleitung zu den konventionswidrig griechisch betitelten „Erotapokriseis des Pseudo-Kaisarios“ auf eine nicht in den „Texten und Untersuchungen “ erschienene Untersuchung zur Überlieferungsgeschichte und gibt nicht einmal eine kurz gefaßte Zusammenstellung der Handschriften ${ }^{49}$. Natürlich kennt die Geschichte der GCS auch mustergültige Editoren, die sich streng an die Konventionen gehalten haben und eine historisch orientierende Einleitung verfaßt haben, die gleichwohl alle unentbehrlichen philologischen und kodikologischen Informationen enthielt: So zum Beispiel Otto Stählin im ersten Band der Clemens-Ausgabe oder in seinen ausführlichen Bemerkungen zu den Fragmenten desselben Autors im dritten

traduction et notes par L. Brésard, H. Crouzel avec la collaboration de M. Borret, SC 375 , Paris 1991, 69.

45 Der Hirt des Hermas, hg. v. M. Whittaker, GCS Die apostolischen Väter I, Berlin ${ }^{2} 1967$ (= 1956), IX-XXVI.

46 H. Chadwick, The New Edition of Hermas, JThS 8, 1957, (274-280) 276-278.

47 Die Pseudoklementinen II. Rekognitionen in Rufins Übersetzung, hg. v. B. Rehm, 2., verbesserte Aufl. v. G. Strecker, GCS Pseudoklementinen II, Berlin 1994, XII-CXXI.

48 Basilius von Caesarea. Homilien zum Hexaemeron, hg. v. E.A. de Mendieta † u. St.Y. Rudberg, GCS.NF 2, Berlin 1997, IX-XIX; dies., Basile de Césarée. La tradition manuscrite directe des neuf homélies sur l'Hexaéméron. Étude philologique, TU 123, Berlin 1980.

49 Pseudo-Kaisarios, Die Erotapokriseis, erstmals vollständig hg. v. R. Riedinger, Berlin 1989, VII-XIV; vgl. ders., Pseudo-Kaisarios. Überlieferungsgeschichte und Verfasserfrage, München 1969. 
Band $^{50}$. Bruno Violet stellte seiner Ausgabe der Esra- und Baruch-Apokalypse eine Gliederung voran ${ }^{51}$, um dann „Textüberlieferung “, „Entstehungs-“ und „Benutzungsgeschichte“ zu behandeln; ganz analog hatte er schon zuvor den vierten Esra herausgegeben ${ }^{52}$. Willem Hendrik van de Sande Bakhuyzen besprach in seiner Ausgabe des Adamantius noch zusätzlich „die Personen des Dialogs“ und gab, was sonst ganz selten geschieht, Hinweise zum Alter der Gliederung des Textes in Sektionen ${ }^{53}$. Ivar Heikel wagte sich auf schwieriges historisches Parkett, als er 1902 in seinem Vorwort zur Konstantin-Vita des Eusebius „die religiöse Anschauung Constantins auf Grund seiner eigenen Schreiben" explizierte - ein seither häufig und durchaus kontrovers verhandeltes Thema ${ }^{54}$. Dafür findet sich in diesem ersten Band der Berliner Eusebius-Ausgabe keine Zeile zum Autor Eusebius, wohl aber in der Neubearbeitung von Friedhelm Winkelmann ${ }^{55}$. Erich Klostermann erläuterte den Lesern seiner Ausgabe des eusebianischen biblischen Ortsnamenkataloges ausführlich „die Arbeit des Eusebius“ als Geograph des Heiligen Landes ${ }^{56}$. Auf diese Weise könnte ich nun noch eine ganze Reihe von praefationes der „Griechischen Christlichen Schriftsteller" rubrizieren; ich setze diese Aufzählung freilich nicht fort, denn der Befund ist deutlich: Eine überwiegende Zahl von Editoren ist dem Modell einer historisch orientierenden praefatio gefolgt - hat also trotz aller Unterschiede im Detail teils recht ausführliche Informationen zum Autor und zu seinem Werk den philologischen und kodikologischen Abschnitten vorangestellt. Dies gilt bis in die jüngste Zeit: Günther Christian Hansen hat seiner Sokrates-Ausgabe selbstverständlich Bemerkungen zu den Quellen und zum Autor wie der Tendenz seines Werkes mitgegeben ${ }^{57}$.

Natürlich gab es auch gelegentlich Streit um praefationes; zumeist entstand er deswegen, weil der Editor und die Kommission unterschiedli-

50 Clemens Alexandrinus, Bd. 1 Protrepticus und Paedagogus, hg. v. O. Stählin, GCS Clemens Alexandrinus I, Leipzig ${ }^{2} 1936$, IX-LXXXIII; Clemens Alexandrinus, Bd. 3 Stromata Buch VII und VIII. Excerpta ex Theodoto - Eclogae propheticae - Quis dives salvetur - Fragmente, hg. v. O. Stählin, GCS Clemens Alexandrinus III, Leipzig 1909, XXIX-LXXXIII.

51 Die Apokalypsen des Esra und des Baruch in deutscher Gestalt, hg. v. B. Violet, Leipzig 1924, IX-XII.

52 Dort folgt freilich eine ,vergleichende Übersicht der alten und der neuen Einteilung des Buches" erst am Schluß: Die Esra-Apokalypse (IV. Esra), 1. Tl. Die Überlieferung, hg. v. B. Violet, Leipzig 1910, 444-446.

53 Der Dialog des Adamantius. ПEPI TH $\Sigma$ EI $\Sigma$ $\Theta E O N ~ O P \Theta H \Sigma ~ \Pi I \Sigma T E \Omega \Sigma$, hg. v. W.H. van de Sande Bakhuyzen, Leipzig 1901, XVI-XVIII.XXIV-XXVI.

54 Eusebius Werke, Bd. 1 Über das Leben Constantins. Constantins Rede an die heilige Versammlung. Tricennatsrede an Constantin, hg. v. I.A. Heikel, GCS Eusebius I, Leipzig 1902, LXXXIII-XC.

55 Eusebius Werke, Bd. 1, 1. Tl. Über das Leben des Kaisers Konstantin, hg. v. F. Winkelmann, GCS Eusebius I/1, Berlin ${ }^{2} 1991$, XLIX-LVII.

56 Eusebius Werke, Bd. 3, 1. Hälfte Das Onomastikon der biblischen Ortsnamen, hg. v. E. Klostermann, GCS Eusebius III/1, Leipzig 1904, IX-XVIII.

57 Sokrates Kirchengeschichte, hg. v. G.Ch. Hansen, GCS.NF 1, Berlin 1995, XLIII-LX. 
che Leitvorstellungen vertraten: Wie wir bereits sahen, war es vor allem das Ideal einer „historisch orientierenden Einleitung“, mit dem vor allem in der Wolle gefärbte Philologen erkennbare Schwierigkeiten hatten. Ich wähle als Beispiel ein Projekt des Unternehmens, das leider immer noch nicht zu einem Abschluß gekommen ist, die Ausgabe der Traditio apostolica, einer wichtigen antiken christlichen Kirchenordnung. Nicht zuletzt aufgrund der Ergebnisse von Eduard Schwartz und Hans Achelis, also von Wissenschaftlern, die der Berliner Ausgabe eng verbunden waren, hatte man beschlossen, diese Kirchenordnung im Rahmen der Werkausgabe des stadtrömischen Theologen Hippolyt erscheinen zu lassen. Angesichts der höchst komplexen Überlieferungsverhältnisse des Textes ${ }^{58}$ erschien aber zunächst keine große synoptische Ausgabe der verschiedenen Versionen im Rahmen der „Griechischen Christlichen Schriftsteller “59 - sie fehlt bis heute -, sondern Editionen der Versionen und Bearbeitungen in den „Texten und Untersuchungen“. So war es jedenfalls geplant. Aufgrund der Schwierigkeiten, in den beiden letzten Kriegsjahren überhaupt noch Texte zum Druck zu befördern, war freilich die Ausgabe der äthiopischen Version, die der Pfarrer Hugo Duensing erarbeitet hatte, 1944 an die Göttinger Akademie gegeben worden und erschien dort tatsächlich kurz nach Ende des Zweiten Weltkrieges ${ }^{60}$. Der nächste geplante Teil war die Ausgabe der koptischen Überlieferung durch Walter Till, die wieder - wie einst geplant - in den „Texten und Untersuchungen“ erscheinen sollte. Als der Text der Ausgabe bereits gesetzt war, wendete sich der Kommissionsvorsitzende Walther Eltester im Oktober 1950 brieflich an die Mitglieder seiner Kommission und schrieb ihnen:

58 Zuletzt: Ch. Markschies, Wer schrieb die sogenannte Traditio Apostolica? Neue Beobachtungen und Hypothesen zu einer kaum lösbaren Frage aus der altkirchlichen Literaturgeschichte, in: W. Kinzig/Ch. Markschies/M. Vinzent, Tauffragen und Bekenntnis. Studien zur sogenannten „Traditio Apostolica“, zu den "Interrogationes de fide“ und zum „Römischen Glaubensbekenntnis“, AKG 74, Berlin/New York 1999, 1-79.

59 Das Protokoll der Kommissionssitzung vom 28.6.1939 hält fest, daß der äthiopische, koptische, der arabische Text sowie eine Rekonstruktion des Griechischen neben dem lateinischen Text in einem Bande des Korpus vereinigt werden sollten und hier also einmal ausnahmsweise die orientalischen Versionen in GCS im Original (und nicht in Übersetzung) geboten werden sollten. Drucktechnische Fragen sind offenbar mit dem Verleger nicht besprochen worden. Der arabische Text sollte von Périer (Les „127 Canons des Apôtres". Texte arabe en partie inédit, publié et traduit en français ... par J. Périer et A. Périer, PO 8/4, Paris 1912 = Turnhout ${ }^{2} 1971,590-622=40-72$ ) übernommen werden, Bearbeiter für die lateinische Überlieferung und die griechische Rekonstruktion waren noch nicht gefunden. In der Sitzung vom 30.7.1948 entschied sich die Kommission dazu, im Korpus zu gegebener Zeit lediglich eine Rekonstruktion der Kirchenordnung in deutscher Sprache herauszubringen und die orientalischen Einzelausgaben in die „Texte und Untersuchungen“ zu übernehmen. Dadurch entstand das Problem mit dem ursprünglich für die große synoptische Ausgabe bestimmten Manuskript Tills, das im folgenden dargestellt wird.

60 Der Aethiopische Text der Kirchenordnung des Hippolyt. Nach 8 Handschriften hg. und übers. v. H. Duensing, AAWG.PH 3. Folge Nr. 32, Göttingen 1946. - Der Autor lebte in Goslar. 
„Die Einleitung von Till ist dürftig. Wenn er auch die Handschriften nicht vor sich hatte, was doch ein ärgerlicher Mangel ist und nun fünf Jahre nach dem Krieg nicht mehr so einleuchtet wie während und kurz nach dem Krieg, so sollte er sie doch wenigstens nennen und kurz ihren Inhalt angeben. Bei der Kompliziertheit der Überlieferung für Hippolyts $\mathrm{K}$ [irchen-]O[rdnung] ist das eine notwendige Hilfe für den Benutzer. Das Datum Mai 1940 für Tills subscriptio kann nicht so bleiben, er muß wenigstens kurz sagen, daß er das Manuskript 1940 abgeschlossen hatte, es aber erst jetzt zum Druck befördern konnte. Zum leichteren Verständnis des ganzen Unternehmens ist doch wohl eine kurze Einführung notwendig. Ich lege einen Vorschlag bei. ... Wer liest übrigens die Korrektur des Koptischen mit: Grapow? Oder Hinze? Ich habe, vertraulich gesagt, das Gefühl, dass Till von dem Inhalt gar nichts versteht, sondern seine Aufgabe rein linguistisch anfaßt“61.

Im Laufe der weiteren Korrespondenz über die Dürftigkeit der Einleitung wurde deutlich, daß Till mit seiner sparsamen Behandlung des handschriftlichen Befundes in Einleitung wie Ausgabe nur den Anweisungen gefolgt war, die ihm der Vorgänger Eltesters, Hans Lietzmann, brieflich im Juni 1940 gegeben hatte ${ }^{62}$; offenbar hatte der schwer erkrankte Berliner Kirchenhistoriker die Probeseiten und das Manuskript selbst auch nicht mehr gründlich durchsehen können. Till hatte im wesentlichen einen älteren Text, den Lagarde ediert hatte, abgedruckt und mit einer deutschen Übersetzung versehen; aufgrund der dürftigen Einleitung mußte Eltester daher seine Kommissionskollegen fragen, ob sie feststellen könnten,

\begin{abstract}
„warum Till seine Aufgabe mit der Wiedergabe des Lagardeschen Textes, der die sahidische Übersetzung wiedergibt, im wesentlichen erschöpft sieht und die bohairische Übersetzung nur vergleichsweise heranzieht. Ist die bohairische Übersetzung soviel jünger und also überlieferungsgeschichtlich soviel wertloser? Ich frage Sie danach, möchte aber meinen, der Herr Herausgeber selbst sollte in seinem Vorwort etwas darüber sagen. Ich vermute, daß darüber bei Lagarde etwas zu finden sein wird, doch sollte Till nicht voraussetzen, daß seinen Lesern diese seltene Ausgabe zur Verfügung steht" ${ }^{63}$.
\end{abstract}

Die Probleme mit Tills praefatio deuteten also in Wahrheit auf Probleme der Textkonstitution. Eltester nennt sie in seinem zweiten Brief an die Kommission im Detail, mutmaßt, daß Till wichtige Handschriften der bohairischen Überlieferung übersehen hat, und schließt: „Die Unsicherheit über solche Fragen finde ich quälend“"64. Till antwortete freilich wider

61 Brief W. Eltester an die Mitglieder der Kommission vom 2.10.1950; zitiert nach maschinenschriftlichen „Auszügen aus dem Schriftwechsel der Kommission btr. Till, Kirchenordnung Hippolyts" (in den Akten der Arbeitsstelle in Berlin [fol. $1^{\mathrm{r}}$ ]).

62 Brief J. Irmscher an W. Eltester vom 16.10.1950; zitiert nach maschinenschriftlichen „Auszügen aus dem Schriftwechsel der Kommission btr. Till, Kirchenordnung Hippolyts“ (in den Akten der Arbeitsstelle in Berlin [fol. $\left.1^{\mathrm{r}}\right]$ ).

63 Brief W. Eltester an die Mitglieder der Kommission vom 23.10.1950; zitiert nach maschinenschriftlichen „Auszügen aus dem Schriftwechsel der Kommission btr. Till, Kirchenordnung Hippolyts“ (in den Akten der Arbeitsstelle in Berlin [fol. $\left.1^{v}\right]$ ).

64 Ebd. 
Erwarten nicht freundlich auf die Bitte, seine praefatio mindestens um ausführliche Bemerkungen zu den Handschriften und der Überlieferungssituation zu erweitern, sondern erklärte, er müsse „leider sagen, daß ich mich dieser Aufgabe durchaus nicht gewachsen fühle“:

„Ich sehe gut ein, daß nun, da der Plan geändert werden mußte und die Übersetzung des koptischen Textes für sich allein herausgegeben wird, eine Einleitung notwendig ist. Doch bin ich gewiß nicht der Mann dazu, sie zu verfassen. Ich verfüge nicht über die nötige Sachkenntnis, habe mich mit dem Gegenstand auch sonst nicht befaßt und hätte eine solche Aufgabe auch niemals übernehmen können. Ich kenne auch die Handschriften nicht, habe nie davon Photos gesehen und weiß auch nicht, wo sie sich gegenwärtig befinden. Gewiß würde es unter den derzeit herrschenden Verhältnissen sehr schwer, wenn nicht unmöglich sein, sie studieren zu können, um eine Beschreibung zu verfassen und eine Kollationierung durchzuführen" 65 .

In seiner brieflichen Reaktion auf diese ernüchternde Antwort stellte nun Walther Eltester, Harnacks zweiter Nachfolger im Amt der Leitung, ausdrücklich die gewohnte Art, in der die Reihe praefationes als „historisch orientierende“ Einleitungen angelegt sehen wollte, zur Disposition und revidierte seine eigenen ursprünglichen Erwartungen:

„Die Einleitung soll ja durchaus nicht auf die historischen Probleme der Überlieferung eingehen, sondern rein philologisch den Tatbestand im Koptischen einsichtig machen. Wir können auf diesen nicht verzichten, wenn er auch so kurz wie möglich erörtert wird. So bliebe denn zu fragen, ob ein zweiter Bearbeiter neben Till gesetzt wird" 66 .

Bleibt zum Abschluß nachzutragen, daß dem Vorschlag Eltesters gefolgt wurde; Johannes Leipoldt trat Till zur Seite, und der Band erschien in erheblich veränderter Gestalt mit einer ausführlicheren Einleitung des Leipziger Religionswissenschaftlers im Jahre $1954^{67}$.

Spätestens wenn man auf die Rezensionen der Bände blickt, wird neben solchen problematischen Einzelfällen wie dem ursprünglichen Manuskript von Tills Einleitung ein gewichtiges Problem vieler praefationes der Reihe deutlich, das Kardinalproblem ihrer Länge. 1903 veröffentlichte Erwin Preuschen seine Edition des Johanneskommentars des alexandrinischen Theologen Origenes ${ }^{68}$. In seiner relativ kritischen Rezension des Bandes

65 Brief W. Till an die Kommission; zitiert nach maschinenschriftlichen „Auszügen aus dem Schriftwechsel der Kommission btr. Till, Kirchenordnung Hippolyts“ (in den Akten der Arbeitsstelle in Berlin [unpaginiert; Blatt $3^{\mathrm{r}}$ ).

66 Brief W. Eltester an die Mitglieder der Kommission vom 22.3.1951; zitiert nach maschinenschriftlichen „Auszügen aus dem Schriftwechsel der Kommission btr. Till, Kirchenordnung Hippolyts“ (in den Akten der Arbeitsstelle in Berlin [unpaginiert; Blatt $\left.3^{\mathrm{r}}\right]$ ).

67 Der koptische Text der Kirchenordnung Hippolyts, hg. und übers. v. W. Till u. J. Leipoldt, TU 58, Berlin 1954, VII-XXII.

68 Origenes Johanneskommentar, hg. v. E. Preuschen, GCS Origenes IV, Leipzig 1903. 
nannte Paul Koetschau nicht nur den Umfang der Einleitung („CVIII Seiten“), sondern bemerkte auch, daß sie „ohne Schaden um mehrere Seiten hätte gekürzt werden können "69. Preuschen hatte sich an die Ratschläge der Kommission gehalten und auf fünfzehn Seiten auch die indirekte Überlieferung abgehandelt, wie sie sich in den Katenen findet: Koetschau warf ihm hier ebenso wie bei der Edition ausgewählter Randglossen einer Handschrift schlampige Arbeit vor ${ }^{70}$. Auf der anderen Seite wurden ausführlichere Einleitungen von Rezensenten durchaus auch gelobt ${ }^{71}$, und Josef Ziegler warf man eine zu knappe praefatio vor: Er habe die Nachwirkung des eusebianischen Kommentars auf Hieronymus und Theodoret nicht berücksichtigt, auch die theologischen Probleme ausgeklammert, damit den Dogmenhistorikern und Patristikern überlassen ${ }^{72}$. Und so hat man in einem Fall jüngst bei einem korrigierten Nachdruck ein für die zweite Auflage radikal gekürztes Vorwort in ganzer Länge von fast einhundert Seiten wiederhergestellt ${ }^{73}$, obwohl man dem Gräzisten, der 1954 die praefatio hatte auf ein Drittel zusammenschrumpfen lassen, seinerzeit durchaus „im ganzen das Lob“ ausgesprochen hat, „,daß er diese Kürzung mit Geschick vorgenommen hat"“74.

Einen Sonderfall der Berliner Kirchenväterausgabe stellen schließlich praefationes dar, deren besondere Gestalt sich durch die historischen Umstände, unter denen die Reihe seit dem Zweiten Weltkrieg produziert wurde, erklärt: Günther Christian Hansen vervollständigte nicht nur die von Joseph Bidez hinterlassene Edition der Kirchengeschichte des Sozomenus, sondern schrieb auch eine ausführliche Einleitung zum Werk, für die ihm keinerlei Vorarbeiten des damals schon längst verstorbenen Ge-

69 P. Koetschau, Rez. GCS Origenes IV, ThLZ 29, 1900, (657-661) 659. - Vgl. den Brief, den U. v. Wilamowitz-Moellendorff an Harnack am 29.11.1904 in dieser Sache schrieb: Text bei J. Dummer, Ulrich von Wilamowitz-Moellendorff und die Kirchenväterkommission der Berliner Akademie, in: J. Irmscher/P. Nagel (Hgg.), Studia Byzantina II, Berlin 1973, (351-387) Brief nr. 9, p. 373f.

70 Koetschau, Rez. GCS Origenes IV (wie Anm. 69), 659 f.

71 E. Gamillscheg, Rez. GCS Eusebius I/1, JÖB 27, 1978, (356-358) $356 f$.

72 W. Lackner, Rez. GCS Eusebius IX, Gn. 72, 1979, (71-77) 72f.

73 Vgl. G.Ch. Hansen, Vorwort zur dritten Auflage, in: Theodoret Kirchengeschichte, hg. v. L. Parmentier, 3., durchgesehene Aufl. v. G.Ch. Hansen, GCS.NF 5, Berlin 1998, V: „Die ausführliche Einleitung Parmentiers hat er (= Felix Scheidweiler, C.M.) auf ein Minimum von 21 Seiten gekürzt, so daß die wissenschaftliche Beschäftigung mit der Kirchengeschichte Theodorets meist auf die Ausgabe von 1911 zuückgreifen mußte“. Vgl. auch die von L. Früchtel für Benutzer von GCS Clemens Alexandrinus II hinzugefügte „Einleitung “: „Da der erste Band der Gesamtausgabe des Clemens Alexandrinus, in welchem Stählin über die Überlieferung referiert hat, nicht in der Hand aller Benützer der vorliegenden Neuausgabe vorausgesetzt werden kann, wird hier eine Zusammenfassung des notwendigsten Materials gegeben, jedoch vielfach in neuer Sicht und auf den jetzigen Stand der Forschung gebracht" (GCS Clemens Alexandrinus II, Berlin ${ }^{4} 1983$, VII Anm. 1).

74 W. Göber, Rez. GCS Theodoret (hg. v. F. Scheidweiler), ByZ 50, 1957, 161f., Zitat 161. 
lehrten zur Verfügung standen ${ }^{75}$. Max Rauer erweiterte und revidierte nicht nur seine Ausgabe der Lukashomilien des Origenes, sondern gestaltete auch das Vorwort durchgreifend $u^{76}$. Albertus Frederik J. Klijns Einleitung zu seiner Übersetzung des lateinischen Textes der Esra-Apokalypse muß man als Supplement und Korrektur zu der im Band nicht abgedruckten Einleitung von Bruno Violet ansprechen ${ }^{77}$. Hans-Martin Schenke begründete mit einem längeren Vorwort im Jahre 1981, warum eine ältere Ausgabe der „Griechischen Christlichen Schriftsteller“ mit Addenda et Corrigenda nachgedruckt und nicht gänzlich neu bearbeitet worden war, nämlich die vierte Auflage des ersten Bandes der „koptischgnostischen Schriften “78. Hans Quecke hat entsprechend auch in seiner Rezension das Gespräch mit diesem Vorwort aufgenommen und gefragt, ob nicht wirklich eine Neubearbeitung des ursprünglichen Textes erforderlich wäre ${ }^{79}$. Ludwig Früchtel hat schließlich in seiner Einführung in die Matthäusschriften des Origenes im dritten Band der Matthäuserklärung eine eingehende Beurteilung und Kritik der lateinischen Überlieferung vorgelegt, die ein deutliches Gegengewicht zur wesentlich positiveren Beurteilung ihres Quellenwertes durch den Co-Editor Erich Klostermann darstellt (freilich ist dieser Beitrag in der zweiten Auflage dann wieder entfallen $)^{80}$.

Ich möchte meine drei Erkundungsgänge mit einem knappen Schlußwort versehen.

\section{Schlußbemerkungen}

Es dürfte trotz unserer Konzentration auf einzelne Ausgaben des Corpus Berolinense und wenige andere Editionen deutlich geworden sein, daß sich praefationes von kritischen Textausgaben vorzüglich dazu eignen, Editions-

75 Vgl. A. Primmer, Rez. GCS Sozomenus, Gn. 39, 1967, (350-358) 351f. - Man könnte hier natürlich auch die posthume Fertigstellung des Vorwortes zu Hippolyt III nach dem Tode von Paul Wendland am 10.10.1915 anführen, vgl. den Brief von U. v. WilamowitzMoellendorff an Harnack vom 13.10.1915 bei Dummer, Ulrich von WilamowitzMoellendorff (wie Anm. 70), nr. 16, p. 382f.

76 Vgl. Origenes Werke, Bd. 9 Die Homilien zu Lukas in der Übersetzung des Hieronymus ... hg. v. M. Rauer, GCS Origenes IX, Berlin 1930, IX-LXII, mit Origenes Werke, Bd. 9 Die Homilien zu Lukas in der Übersetzung des Hieronymus ... hg. u. in zweiter Auflage neu bearb. v. M. Rauer, GCS Origenes IX, Berlin 1959, VII-LXIV.

77 Die Esra-Apokalypse (IV. Esra), nach dem lateinischen Text unter Benutzung der anderen Versionen übersetzt und hg. v. A.F.J. Klijn, Berlin 1992, XI-XXXV.

78 GCS Koptisch-Gnostische Schriften, 1. Bd. Die Pistis Sophia. Die beiden Bücher des Jeû. Unbekanntes altgnostisches Werk, hg. v. C. Schmidt, 4., um das Vorwort erweiterte Auflage hg. von H.-M. Schenke, Berlin 1981.

79 H. Quecke, Rez. Koptisch-gnostische Schriften I, ThRev 79, 1983, $214 f$.

80 Origenes Matthäuserklärung III. Fragmente und Indices, hg. v. E. Klostermann, GCS Origenes XIII, 2. Hälfte Gesamtregister v. L. Früchtel, Berlin 1955, 23-52; vgl. die Bemerkungen v. U. Treu in der zweiten Auflage, Berlin 1968, V. 
reihen und einzelne Ausgaben in ihrem Rahmen zu charakterisieren, Tendenzen zu bestimmen. Das Berliner Kirchenväterkorpus trat mit einer kleinen Revolution an; es gab seinen Ausgaben deutsche praefationes bei und gestaltete den Apparat ebenfalls in dieser Sprache. Es verpflichtete seine Bearbeiter auf eine „historisch orientierende“ Einleitung, begriff also nicht nur die Funktion der Ausgabe insgesamt in einem weiten kulturgeschichtlichen Horizont, sondern legte auch ihre einzelnen Bände so an. Nun muß man sich als eines der Kommissionsmitglieder, die heute die Verantwortung für diese Reihe tragen, fragen, ob der entsprechende Impetus, dem die Ausgabe seit 1891 dienen wollte, damals schon konsequent umgesetzt wurde oder jedenfalls heute konsequentere Umsetzungen möglich sind. Die Stichworte, die hier zu nennen sind (mehr als Stichworte können es an dieser Stelle freilich nicht sein), lauten: Müßten nicht, wenn schon die praefationes und der Apparat in deutscher Sprache angelegt sind, auch deutsche Übersetzungen beigegeben werden? Kann man sich nicht auch englische Einleitungen und ebensolche Übersetzungen vorstellen? Schon im Jahre 1907 fragte die Hinrichs'sche Buchhandlung die Kirchenväter-Kommission, ob nicht „,von den ,Griechischen christlichen Schriftstellern der ersten drei Jahrhunderte' deutsche Übersetzungen veranstaltet würden“, und nannte dies ein „wirkliches wissenschaftliches Bedürfnis“81. Harnack nannte damals zwar Übersetzungen für einzelne Bände „- nicht für das gesamte Corpus - wünschenswert“ und regte an, zugleich „einen Commentar ins Auge zu fassen " 82 , aber seinem Kommissionskollegen, Mommsens Nachfolger Otto Hirschfeld, lag daran, daß solche Übersetzungen nicht als offizielles Projekt der Kommission firmierten ${ }^{83}$. Und so sind bis heute wichtige Texte der Reihe wie Eusebs Praeparatio evangelica noch ohne deutsche Übersetzung. Eine weitere Frage stellt sich angesichts der praefationes, die wir behandelt haben: Müßten die verschiedenen Nachfolgeinstitutionen der Berliner Kirchenväterkommission nicht viel deutlicher auf Harnacks alter Idee der „historisch orientierenden“ praefatio bestehen, zumal die Kenntnisse des historischen Umfeldes der kaiserzeitlichen und spätantiken Christenheit in den letzten Jahrzehnten gewiß nicht sonderlich zugenommen haben? Und eine dritte Frage stellt sich

81 Brief J.C. Hinrichs'sche Buchhandlung an die Kommission vom 13.2.1907, Blatt 1 (Archiv der BBAW, Akten der Kirchenväter-Kommission, Bd. 2 1903-1930, Blatt 99').

82 Ebd.; Harnack nennt in seinem Reskript vier Bände, über deren Übersetzung nachgedacht werden sollte: Eus., h.e.; v.C.; Clem.Al., str.; Or., Cels. - Alle diese Texte wurden im Rahmen der „Bibliothek der Kirchenväter“ übersetzt, wobei Paul Koetschau in seiner Übersetzung der Schrift des Origenes „eine Anzahl textkritischer Bemerkungen, die zur Ergänzung und Berichtigung meiner Ausgabe dienen sollen", beifügte (BKV Origenes II, 1926, XVI). Auch die „Stromateis“ übersetzte ihr Editor im Berliner Korpus, Otto Stählin.

83 Zu Hirschfeld vgl. die Nachweise bei St. Rebenich, Theodor Mommsen und Adolf Harnack. Wissenschaft und Politik im Berlin des ausgehenden 19. Jahrhunderts, Berlin 1997, 43f. 
angesichts des Stichwortes „Informationszeitalter“ im Titel des Symposiums: Welche Standards gelten eigentlich für die praefationes von elektronischen Textausgaben? Sind hier die bisherigen Alternativen hinfällig, weil im Internet alle Versionen gemeinsam unter verschiedenen Adressen publiziert werden können?

\section{ABSTRACT}

Taking as example different editions of the GCS, the Viennese CSEL and the SC, the article shows in which types of praefationes the different introductions of the editions can be grouped. The practice originally used in Berlin differs from the Viennese model and traces back to the "Apostolic Fathers" from Lightfoot. Specific attention is given to the language of the praefatio. Finally, some problematic cases are dealt with where the difficulties of an edition can be shown already with the praefatio. 